\title{
The Ethical, Legal and Human Rights Concerns Raised by Licensing HIV Self-Testing for Private Use
}

\author{
Lucy Allais • Francois Venter
}

Published online: 18 June 2014

(c) Springer Science+Business Media New York 2014

\begin{abstract}
We argue that there are no compelling ethical grounds for not allowing the sale of HIV self-tests to the public, so long as reasonably robust protections are in place to protect against coerced testing, and so long as the ease of use of the test is validated carefully in each country in which it is used, with attention to information about linkage to treatment, social and psychological support. The tests are not likely to be harmful in a way that justifies restricting people's access to them, and have plausible benefits. Whether and how self-testing should be used in public health programs will depend on complex policy questions concerning priorities, efficacy and cost.
\end{abstract}

Keywords Ethics - Human rights - Coercion - HIV self testing

\section{Introduction}

HIV self-tests are currently licensed for sale to the public in some countries, including the UK and USA, but not in others [1]; the question of whether there are ethical concerns with respect to licensing these tests requires attention. Our focus in this paper is specifically with possible ethical objections to licensing HIV tests for off-the-shelf sale for purchase by individuals who could then test themselves.

\footnotetext{
L. Allais $(\bowtie)$

University of the Witwatersrand and Sussex University,

Johannesburg, Gauteng, South Africa

e-mail: Lucy.allais@wits.ac.za

F. Venter

Wits Reproductive Health and HIV Institute, University of the Witwatersrand, Johannesburg, South Africa

e-mail: fventer@wrhi.ac.za
}

The reason for this focus is that considering the private purchase of HIV self-tests highlights the specific concerns raised by this new form of HIV testing: whether there are ethical problems, in particular, with an intervention that makes it possible for individuals to choose to test themselves, and to carry out such tests without involvement of any health care practitioners. HIV self-tests are reliable and involve minimal physical risks [2-6]. By enabling individuals to find out their HIV status without having to go to a medical provider, HIV self-tests have the potential to extend people's choices and to expand knowledge of HIV status. However, beneficial medical interventions can involve some risks and may also be associated with indirect harms and psychological and societal harm. We assess these concerns and argue that they are not sufficient to justify restricting individuals from accessing the HIV selftests themselves, so long as self-testing can provide validated and comprehensible information about accessing treatment as well as psychological and social support [7], a standard that applies to all forms of diagnostic self-testing.

In assessing ethical concerns raised by the use of HIV self-testing (HIVST) it is important to separate two distinct questions: one, whether HIV self-tests should be licensed for private sale so that an individual who wants to test themselves for HIV can do so without needing to go to a medical practitioner or testing center, and two, whether HIV self-tests should be provided by the state or otherwise made use of in state or donor-funded public health programs or to realize public health goals. The first question concerns the ethical grounds for restricting and controlling the access individuals have to a medical intervention. The second concerns the effective use of public funds and the best ways of promoting public health. It is important to keep these distinct, so that worries falling under the second question are not mistakenly taken as objections to the first. 
Concerns raised about the use of HIVST at the WHOsponsored self-testing workshop in Geneva [2] included the worry that rolling out HIVST would wrongly divert priorities away from other areas and that self-testing was a 'middle-class' model based on the assumption of individuals having sufficient disposable income to buy the test, which is unrealistic in poorer communities in sub-Saharan Africa. These are legitimate concerns, but it is clear that they fall under our second question: how should HIVST be used in realizing public health goals? These concerns do not provide a reason to think that individuals should not be allowed to buy themselves an HIV test and test themselves. That a medical intervention is not a priority for realizing public health goals or the optimal instrument for public health programs is not a reason to restrict individuals from choosing to use it for themselves. On the other hand, coherent ethical objections to the first question would mean that the second question, concerning the extension of this form of testing in broader public programs, should not be even considered. This means that even for those whose concern is primarily with public health priorities our first question is important. This ethical question is logically prior to questions concerning the use of HIVST in public health programs; it is an important and timely question, since many countries do not currently license HIVST.

In licensing a medical test or treatment for sale to the public the state takes some responsibility for it. This means that the product has to meet higher standards than do products which are available for sale to the public without this official certification or authorization. At the same time, a standard assumption about human rights in liberal democracies is that the state is not generally entitled to restrict individuals' making life choices, including health choices, so long as their choices do not wrongfully harm others. Once a medical test has reached the relevant standards of reliability and safety, the burden of proof required to justify restricting people from accessing it themselves is high. Grounds for restriction are provided where, despite being reliable and potentially beneficial, a medical intervention also has serious potential dangers. There is significant variation across countries with regard to which health interventions are controlled by professionals and which drugs require prescriptions (for instance antibiotics and the oral contraceptive pill are freely available over the counter in some countries but not in others, methadone is provided as a heroine substitute in the UK but is illegal in Russia) and the legal frameworks determining which drugs we can access ourselves reflect some accidents of history (think of alcohol). However, clear grounds for caution in allowing individuals to access certain medical interventions on their own are provided by the interventions' being potentially harmful in some specific ways. For example, some medicines are potentially addictive, some have severe side effects, some need to be administered by a skilled professional, and some have effects on other individuals or communities. Each of these factors could provide reasons for not allowing individuals to buy and apply a medical intervention themselves. We need to consider, therefore, whether any similar reasons apply in the case of HIVST.

\section{What are the Benefits?}

Before considering the possible harms of licensing HIVST for individual access it is important to note significant possible benefits of such tests. In general, allowing people to manage their health, including by finding out important information about their health for themselves, is a good thing. Given the nature of its infectiousness and treatment, knowledge of HIV status is especially significant and HIV programs regard increasing knowledge of HIV status as important $[8,9]$. It follows from this that any non-harmful measure with the potential for making a contribution to this should be welcomed. Further, while there may be places in which even the cheapest self-tests will be too expensive for most people to access, this is not true in the developed world and is also not true in some of the countries in which HIV is endemic. For example, there will be very many people who can afford to buy such a test in a middleincome country such as South Africa, which has a per capita net income of almost USD \$8,000 [10]. In some communities and some workplaces (such as medical clinics) it is difficult for people to access testing without coworkers or community members knowing they are doing so, and perhaps even knowing their results, and lack of trust in confidentiality may discourage people from accessing testing sites. Privately purchased over-thecounter self-testing gives these people an important option. There is evidence that men access testing centers less than do women (a concern raised by more than one talk presentation at the WHO workshop in Geneva, 2), and are significantly under-tested as a group [11-13]. Licensing self-testing may help contribute to addressing this problem, and therefore to expanding testing in a large and important group. Finally, access to HIVST may contribute to the normalization of HIV and HIV testing.

\section{Are There Harms to the Individual?}

If HIV self-tests were difficult to use or difficult to interpret, so that without the intervention of a medical professional individuals would be reasonably likely to use them incorrectly or to get the wrong result, this would provide reason for the state not endorsing them for sale to the 
public. The sensitivity, accuracy and ease of use of the tests are not issues to be determined by ethicists, and have been changing due to advances in technology and packaging; there is good reason to think the test currently licensed in the USA and elsewhere is safe [2-6]. However, a contextspecific validating process is necessary in each country in which the test is used, including outlining linkage to treatment, social and psychological support [7].

The test itself has no physical harms or side-effects. However, it might have psychological effects and this might be taken to suggest that HIV testing should not happen without counseling or some form of supervision. Grounds for routinely linking HIV testing to counseling include the possibility of linking a person with a positive result to access to treatment, using the test to provide information about safer sex practices, helping people disclose to their partners (particularly in relationships where there may be violence), and helping to prevent serious negative psychological harms of a positive result, such as depression and suicide [8, 14]. It should be noted that in many actual HIV testing programs counseling is poor, as is linkage to treatment, and that in many countries patients access testing privately without counseling, and are given tests without counseling by insurance companies [15, 16]. We should be careful of comparing self-testing with an imaginary ideal of perfect counseling rather than with the counseling that is likely to occur, and is occurring, in actual programs, particularly if the comparison is being used to motivate restricting access to self-testing. However, against this argument, if it were plausible that HIV testing without counseling risked serious harms, the fact that existing counseling is poor would not justify allowing self-testing, but would rather call for urgent attention to improving existing counseling.

The most compelling reason for thinking that self-testing without counseling could be harmful is the possible negative psychological impact of a positive diagnosis, which may include depression and risk of suicide. Counseling can also play a crucial role in helping people disclose to families. However, the idea that depression is dramatically more likely to follow a positive result in the absence of the short post-test counseling session is, in our view, implausible and psychologically naïve: in all medical settings an unwelcome diagnosis, such as cancer, or an incurable or untreatable illness, may take time to sink in. Similar points apply to support with disclosure to partners, and for dealing with violent partners. Of course, counseling need not only take the form of a short conversation that accompanies the actual test, but rather can involve ongoing support groups, information about shelters from domestic violence, and other forms of follow-through. However, these services can also be available to those who make use of self-testing, so long as the packaging accompanying
HIV self-tests provide information about accessing such services, including phone numbers for help lines [2]. Support services can also make use of other formats that do not involve face-to-face contact, such as the delivery of messages, information and support over mobile phones; HIVST may provide an opportunity to pay attention to developing such possibilities. All forms of testing, including self-testing, should provide information about the ongoing possibility of accessing psychological and social support, as well as treatment.

It is important to see that the question of whether testing without counseling is unacceptable is separate from the question of whether counseling can provide important benefits; there are many reasons to think that it does [14]. The central understanding of human rights in liberal democracies holds that when the state restricts significant individual choices the standard of justification is not just that the restriction might provide benefits, but rather, it must be shown that the alternative is so seriously harmful that the state has a duty to protect individuals from it. For example, there may be benefits associated with combining pregnancy or diabetes tests with counseling, but the fact that testing without counseling would mean testing without these benefits would not justify restricting people from accessing such testing themselves. Consider having a policy of requiring women to have counseling about family planning before allowing them to buy a pregnancy test. Such a ruling would be inappropriately intrusive and paternalistic. In order to show that the absence of counseling is a reason for not allowing the private purchase of HIV self-tests it must be shown not just that counseling might have benefits, but that testing without counseling is positively and seriously harmful. For example, it would need to be shown not just that counseling might help link people with treatment, but that self-testing will make people positively unlikely to access treatment, or positively and significantly more likely to have unsafe sex than not being allowed to test themselves would. Neither of these results seems plausible, and compelling evidence for them would need to be provided to justify the restriction.

\section{What About Harms to Others?}

We have considered the possible harms to individuals of use of HIV self-tests on themselves. However, a separate ground for ethical and human rights concern is whether such tests could be used in coercive ways that are harmful to vulnerable people. Two areas of particular concern are employers and abusive family members, who could conceivably purchase HIV self-tests and bully their employees, family members or partners to test. This is a serious concern. However, it is not clear that it provides grounds for 
restricting private purchase of HIVST. We place restrictions on individuals' autonomy when it comes to harming others; our fundamental right to choose how to live our lives for ourselves does not include the right to wrongfully harm others. But restricting people from harming others is very different from forbidding access to everything which might conceivably be used to harm others, which is impossible (aspirin, pregnancy tests, and steak knives, for example, could all be misused and used in abusive ways). It is important here to be clear about the logic of the argument and the burden of proof. To justify restricting individuals' access it is not enough that the tests can used coercively; rather, we need compelling evidence for thinking that access to self-testing would create or seriously exacerbate abusive relations in ways that are in tension with the state's duty to protect vulnerable groups, and the burden of proof is on those who want the restriction. Of course, this by no means implies that paying attention to abuses, working on ways to prevent them, and defending the law, are unimportant, and should not be a focus of policy makers and activists. Further, in countries in which there is no legal framework protecting people from, for example, being forced by their employers to test, there may be reasons to be careful about promoting selftesting. However, holding HIV self-tests to a standard which requires showing that they will never be used abusively, or that they are the optimal instrument for promoting public health or providing the highest possible standard of care, treats HIV self tests in a way that is different to other medical tests and interventions; in our view, this is an example of HIV exceptionalism [16].

\section{What About the Law?}

We have looked at ethical and human rights-based concerns. There may also be concern about the legal implications of knowing your HIV status, since some countries treat having unprotected sex while knowing that you are HIV positive as criminal if undisclosed, and some even where it is disclosed and both parties consent, or where treatment has rendered the person non-infectious [17]. In our view, many of these laws are discriminatory and inappropriately single out HIV, and should be strongly opposed. We do not think there is reason to regard an HIV positive person who discloses her HIV status to her sex partner as recklessly or wrongfully endangering her sex partner more than does someone who does not know her HIV status (on the contrary, there are contexts in which the latter may be more reckless), and it is highly questionable whether the criminal law is a reasonable way of dealing with either $[17,18]$. While such laws are a concern, it is not clear that self-testing, as opposed to conventional HIV testing, raises particular concerns, since people are equally liable however they find out their status. However, in countries which have such laws, cautionary information about them should be provided together with all forms of testing, including self-testing.

\section{Conclusions}

Many questions remain to be answered about whether and how HIVST should be used in public health programs, and questions of resource allocation and policy efficacy always have significant ethical, social, and political implications. However, as important as these issues are, they are not a function of the specific innovative feature of this new form of testing: individuals being able to test themselves. In our view there are no ethical objections to allowing people to access and carry out validated and supported HIV testing themselves in countries with appropriate protective laws. Licensing such tests has a potential to empower some people, and to make a contribution to the important goal of widening knowledge of HIV status.

\section{References}

1. Alcorn A. Legal barrier to self-testing for HIV in UK to be lifted. NAM AIDSMAP [cited 2013 Oct 14]. Available from: http:// www.aidsmap.com/Legal-barrier-to-self-testing-for-HIV-in-UKto-be-lifted/page/2731557/.

2. World Health Organization (WHO). Report on the first international symposium on self-testing for HIV: the legal, ethical, gender, human rights and public health implications of HIV self testing scale up. Geneva: WHO; Apr 8-9, 2013 [cited 2013 Oct 14]. Available from: http://apps.who.int/iris/bitstream/10665/ 85267/1/9789241505628_eng.pdf.

3. Pant Pai N, Sharma J, Shivkumar S, Pillay S, Vadnais C, et al. Supervised and unsupervised self-testing for HIV in high- and low-risk populations: asystematic review. PLoS Med. 2013;10(4):e1001414. doi:10.1371/journal.pmed.1001414.

4. Choko AT, MacPherson P, Webb EL, Ball H, Sambakunsi R, Mdolo A, Makombe SD, Desmond N, Hayes R, and Corbett EL. One year outcomes following community-based HIV self-testing: a prospective study in Malawi, Abstract 147. Presented at Conference on Retroviruses and Opportunistic Infections, Boston. 3-6 Mar 2014.

5. Asiimwe S1, Oloya J, Song X, Whalen CC. Accuracy of unsupervised versus provider-supervised self-administered HIV testing in Uganda: a randomized implementation trial. AIDS Behav. Apr 2014 [cited 2014 May 27]. Available from: http:// www.ncbi.nlm.nih.gov/pubmed/24691923.

6. United States Food and Drug Administration (US FDA). OraQuick ${ }^{\circledR}$ In-home HIV Test summary of safety and effectiveness. Silver Spring, MD: US FDA; 2012 [cited 2014 May 4]. Available from: http://www.fda.gov/downloads/BiologicsBloodVaccines/Blood BloodProducts/ApprovedProducts/PremarketApprovalsPMAs/ UCM312534.pdf.

7. Krause J, Subklew-Sehume F, Kenyon C, Colebunders R. Acceptability of HIV self-testing: a systematic literature review. BMC Public Health. 2013;8(13):735. doi:10.1186/1471-2458-13735. 
8. World Health Organization (WHO). Guidance on provider-initiated HIV testing and counselling in health facilities. Geneva: WHO; 2007 [cited 2014 Jan 4]. Available from: http://whqlibdoc. who.int/publications/2007/9789241595568_eng.pdf

9. South African National Department of Health. National HIV counselling and testing policy guidelines, Pretoria: National Department of Health; 2010 [cited 2014 May 21]. Available from: http://www.uj.ac.za/EN/CorporateServices/ioha/Documenta tion/Documents/hct_policy_guidelines\%202010.pdf

10. The World Bank. Data South Africa. World Bank Group; 2014 [cited 2014 Jan 5]. Available from: http://data.worldbank.org/ country/south-africa

11. The Joint United Nations Programme on HIV/AIDS (UNAIDS). UNAIDS report on the global AIDS epidemic. Geneva: UNAIDS; 2013: p.80 [cited 2014 Jan 4]. Available from: http://www. unaids.org/en/media/unaids/contentassets/documents/epidemiology/ 2013/gr2013/UNAIDS_Global_Report_2013_en.pdf

12. Ngảngả A, Waruiru W, Ngare C, Ssempijja V, Gachuki T, Njoroge I, Oluoch P, Kimanga DO, Maina WK, Mpazanje R, Kim AA, KAIS Study Group. The status of HIV testing and counseling in Kenya: results from a nationally representative population-based survey. J Acquir Immune Defic Syndr. 2014;66(Suppl 1):S27-36. doi:10.1097/QAI.0000000000000102.
13. Venkatesh KK, Madiba P, De Bruyn G, Lurie MN, Coates TJ, Gray GE. Who gets tested for HIV in a South African urban township? Implications for test and treat and gender-based prevention interventions. J Acquir Immune Defic Syndr. 2011;56(2):151-65. doi:10.1097/QAI.0b013e318202c82c.

14. Fonner VA, Denison J, Kennedy CE, O'Reilly K, Sweat M. Voluntary counseling and testing (VCT) for changing HIV-related risk behavior in developing countries. Cochrane Database Syst Rev. 2012;9:CD001224. doi:10.1002/14651858.CD001224.pub4.

15. Rosen S, Fox MP. Retention in HIV care between testing and treatment in sub-Saharan Africa: a systematic review. PLoS Med. 2011;8(7):e1001056. doi:10.1371/journal.pmed.1001056.

16. Richter M, Venter WD, Gray A. Home self-testing for HIV: AIDS exceptionalism gone wrong. S Afr Med J. 2010;100(10): 636-42.

17. Weait M. Intimacy and responsibility: the criminalization of HIV transmission. Oxon: Routledge-Cavendish; 2007.

18. Jürgens R, Cohen J, Cameron E, Burris S, Clayton M, Elliott R, et al. 10 Reasons to oppose the criminalization of HIV exposure or transmission; New York: Open Society Institute; 2008 [cited 2013 Oct 19]. Available from: http://www.opensocietyfoundations.org/ sites/default/files/10reasons_20081201.pdf. 